\title{
IN MEMORIAM: VLADIMIR VOEVODSKY
}

1966-2017

Vladimir Voevodsky was an algebraist who made enormous contributions to algebraic geometry and to the foundations and formalization of mathematics. He died suddenly of an aneurysm in September, 2017, at home in Princeton, New Jersey, where he was a faculty member in the School of Mathematics at the Institute for Advanced Study. Born in Moscow in 1966, Voevodsky attended Moscow State University and received his Ph.D. at Harvard in 1989-1992 under David Kazhdan.

He had a special talent for bringing techniques from homotopy theory to bear on concrete problems in other fields, which he put to good use his entire life. As an undergraduate he wrote an article with Kapranov aiming to realize a program of Grothendieck involving $n$-groupoids; in the decade after that he applied topology fruitfully to algebraic geometry; and after that he applied topology ingeniously to the problem of formalization of mathematical proofs.

His creative drive had fully blossomed when, in 1996, he announced a proof of an algebraic statement concerning the cohomology of fields, the Milnor Conjecture, and laid the groundwork for the subsequent solution of the Bloch-Kato Conjecture, a generalization of it. More importantly, he revealed a close approximation to the theory of mixed motives of algebraic varieties long dreamed of by Alexander Grothendieck. His work, parts of which were jointly conceived and published with Andrei Suslin and Fabien Morel, fleshes out the details of a world where algebraic varieties and topological spaces live in perfect harmony. In addition, he pioneered the fruitful use of certain Grothendieck topologies, those where the representable presheaves are not necessarily sheaves. For his work he received the Fields Medal in 2002. In 2011 he published a proof of the Bloch-Kato Conjecture, which uses work of Rost. The field of motivic homotopy theory, which he founded, has remained active and vigorous.

In 2002, Voevodsky started to look into formalization of mathematics, and that work occupied him for the rest of his life. His work there could eventually be viewed as even more important than his work in algebraic geometry, because of its potential to affect the way mathematicians conduct their daily research. He worked conscientiously until his death to hasten the day when mathematicians will no longer need to worry about having made 
a mistake in yesterday's work and referees of journal submissions will no longer need to verify correctness of arguments.

In 2006 Voevodsky gave credit to the articles of Makkai for convincing him that "dependent type systems are exactly what we need in order to formalize categorical thinking". Moreover, his understanding of the topological form of a principle he later called univalence was already in place, as was his idea that soundness of the system would be demonstrated by a model whose objects are topological spaces. By 2010 his ideas had crystallized sufficiently that he was able to use his past experience as a computer programmer to create a repository of proofs of theorems he called Foundations, formalized in the formal language provided by the proof assistant Coq-it establishes the foundation, presents the method for encoding informal mathematics in it, and reveals the new mathematical landscape that one encounters there.

To clarify that terminology, consider set theory: its formal language is first order logic; its foundation consists of the notions of set and set membership and the Zermelo-Fraenkel axioms; the encoding of informal mathematics depends on certain conventions, such as the definition of equality of sets, the construction of ordered pairs and the proofs of their properties, the definition of functions as sets of ordered pairs, and the definition of the natural numbers and its operations, including the usual convention that $3:=$ $\{0,1,2\}$. One problem with set theory as a language for formalization is that theorems cannot generally be transported between equivalent situations. For example, the proposition $2 \in 3$, which holds in the natural numbers, yields something false when transported to the equivalent set of non-negative integers, as the integers are traditionally implemented as certain sets of pairs of natural numbers. The landscape brought into view by the adoption of set theory as a foundation is the same as that visible in informal mathematics, which easily accommodates the sets of set theory as the well-founded rooted trees with only one automorphism. Voevodsky emphasized this point of view by introducing the term $Z F$-structure for those trees and by referring to them instead of the sets of set theory.

Now consider Voevodsky's Univalent Foundations and compare it to set theory. Its formal language is based on the type theories introduced by Martin-Löf, whose basic objects are types and their elements. Included in the language are functions, pairs, finite types, natural numbers, a sequence of universes, and equality. The main novelty of Martin-Löf's type theory is that for elements $x$ and $x^{\prime}$ of a type $X$, the equation $x=x^{\prime}$ yields a type, the equality type, or the type of identifications of $x$ with $x^{\prime}$. The notion of proposition is not part of the formal language.

Voevodsky's chosen encoding of informal mathematics in the formal language starts as follows. Building on the well-established intuitionist Curry-Howard correspondence, Voevodsky defined a proposition to be a type any two elements of which are equal (as had been done as early as 2002 [Awodey \& Bauer]); propositions and their elements are used to encode the informal notions of propositions and their proofs. Then he defined a set to be a type $X$, all of whose equality types $x=x^{\prime}$ are propositions. A subtype of a type $X$ defined by a family $x \mapsto P(x)$ of propositions is encoded by the 
type of pairs $(x, p)$, where $p$ is a proof of $P(x)$. A function from one type to another is called an equivalence if each of its fibers has exactly one element.

The foundation of the system consists of a single axiom, the Univalence Axiom. If $X$ and $Y$ are two types living in the universe $U_{n}$, then we can consider the equality type $X=Y$ and the type $X \simeq Y$ of equivalences from $X$ to $Y$. Voevodsky's Univalence Axiom asserts that the natural map $(X=Y) \rightarrow(X \simeq Y)$ is an equivalence. The inverse of that equivalence is used to promote equivalences to equalities, thereby providing automatic transport of theorems between equivalent situations. The law of excluded middle and the axiom of choice are acceptable, too, and, since the language is intuitionistic, it is easy to observe appeals to them, since they are explicit.

Now comes the hard part: establishing enough facts about the chosen encoding to see that it "works". Amazingly, Voevodsky proved and formalized hundreds of them (ignoring resizing issues) in just a few months in 2010 - they constitute his Foundations repository and are a major achievement. Before stating some of them, we present the point of view Voevodsky adopted in his approach to them.

One may draw the following analogy between type theory and topology. Given a type $X$ and elements $x$ and $x^{\prime}$,one may have two elements $p$ and $p^{\prime}$ of the type $x=x^{\prime}$; one may in turn consider whether there is an element $h$ the type $p=p^{\prime}$, and so on. By analogy, one may have a space $X$, points $x$ and $x^{\prime}$ of $X$, paths $p$ and $p^{\prime}$ from $x$ to $x^{\prime}$, and a homotopy $h$ from $p$ to $p^{\prime}$, and so on.

The analogy appears in 2009 [Awodey \& Warren] as the basis for a model in Quillen model categories of the inference rules of a form of type theory that includes equality types, and which models families of types as fibrations. That model in turn was inspired by a 1994 model in groupoids [Hofmann \& Streicher], which implies that it is not provable that every type is a set. Their model was preceded by a 1991 model in groupoids, but without identity types [Lamarche].

The analogy motivates an alternative reading of the language of type theory, where one thinks of a type as a space, an element of a type as a point of that space, and an element of the type $x=x^{\prime}$ as a path from $x$ to $x^{\prime}$. It was with this reading foremost in mind that Voevodsky was able to transfer many facts from homotopy theory to type theory-indeed, one of his first lines of code renames the equality type of Coq to paths.

Mirroring the notion of homotopy truncation level in topology, Voevodsky defines by induction on a natural number $n$ the property that a type $X$ has $h$ level (at most) $n$, as follows: (1) $X$ has $h$-level 0 if it has exactly one element; (2) $X$ has $h$-level $n+1$ if for any elements $x$ and $x^{\prime}$ of $X$, the type $x=x^{\prime}$ has $h$-level $n$. The types of $h$-level 1 are the propositions, and the types of $h$-level 2 are the sets.

The notion of $h$-level provides a unifying principle for the results of the Foundations repository, which include the following: the type of functions from one set to another is a set; a subtype of a set is a set (call it a subset); the type of all subsets of a set is a set; whether a type is a proposition, is a proposition; whether a type is a set, is a proposition; equivalences have 
inverse functions that are equivalences; whether a function is an equivalence, is a proposition; the type of natural numbers and the finite types are sets; a type equivalent to a proposition is a proposition; a type equivalent to a set is a set; propositions that imply each other are equivalent; equivalent propositions are equal; subsets defined by equivalent predicates are equal; and two functions whose corresponding values are all equal, are equal.

One then sees, for example, that a feasible way to encode the algebraic notion of group in the $n$-th universe $U_{n}$, say, is as a 10 -tuple

$$
\left(G, e, i, m, \alpha, \lambda, \rho, \lambda^{\prime}, \rho^{\prime}, \imath\right),
$$

where $G$ is a type appearing as an element of $U_{n}, e$ is the identity element of $G, i: G \rightarrow G$ is the inverse function, $m: G \times G \rightarrow G$ is the multiplication function, $\alpha$ is a proof of associativity, $\lambda$ is a proof of the left unit law, $\rho$ is a proof of the right unit law, $\lambda^{\prime}$ is a proof of the left inverse law, $\rho^{\prime}$ is a proof of the right inverse law, and $l$ is a proof that $G$ is a set. Since proofs of a proposition are equal to each other, there is no way to get a different group by giving a different proof of one of the six properties, and that's in accordance with standard mathematical practice. Let Group denote the type of all such tuples.

Similarly, if $G$ and $H$ are two groups, then they are both elements of the type Group and we may consider the equality type $G=H$. A consequence of the Univalence Axiom is that the natural map from $G=H$ to the type of isomorphisms from $G$ to $H$ is an equivalence. If $G$ and $H$ are isomorphic in more than one way (which is usually the case) then it follows that $G=H$ has more than one element. Thus the equation $G=H$ is a type that is not a proposition, and the type Group is not a set. However, it's almost as good: $G=H$ is a set, because the type of isomorphisms between two groups is a set.

(In set theory it is also true that the class of all groups is not a set, but that is an issue related to size, and we don't evade that issue in type theory, either, for Group lives in the universe $U_{n+1}$. What we mean when we say that Group is not a set is that one of its equality types is not a proposition.)

The discussion above shows that Group is of $h$-level 3. Other inhabitants of $h$-level 3 include the classifying type $B G$ of a group $G$, whose loop space $*=*$ is the group $G$; one implements $B G$ as the type of $G$-torsors, and one sets $*$ to be the trivial torsor. The classifying type $B \mathbb{Z}$ is a type that plays the role of the topological circle, $S^{1}$. A new field, synthetic homotopy theory, proves analogues of theorems of homotopy theory and algebraic topology in this landscape, including the computation of some homotopy groups of spheres, such as $\pi_{n} S^{n}=\mathbb{Z}$ and $\pi_{4} S^{3}=\mathbb{Z} / 2 \mathbb{Z}$, the Freudenthal suspension theorem, and the Blakers-Massey connectedness theorem. Only the theorems of topology admitting the most generality can be proved synthetically, for Martin-Löf's type theory is Spartan and was designed without this goal in mind.

Motivated by the type Group above, we see that a natural definition of category in Univalent Foundations would not include a requirement that the type of objects of the category be a set; it is also natural to add a 
univalence condition, often satisfied in practice: for any two objects $X$ and $Y$ of the category $\mathcal{C}$, the natural map from the type $X=Y$ to the type of isomorphisms from $X$ to $Y$ should be an equivalence. The inverse map of that equivalence allows isomorphisms to be promoted to equalities, and thus allows transport of properties between isomorphic objects. This convention fulfills a long-time dream of category theorists: a language where all concepts expressible in the language are invariant under isomorphism of objects and under equivalence of categories.

Voevodsky viewed $h$-level 1 as the natural home for element-level mathematics, $h$-level 2 is the natural home for set-level mathematics, $h$-level 3 is the natural home for category-level mathematics (where the objects have symmetry), and so on. He regarded the $h$-level hierarchy of types and thus of mathematics itself to be one of his most fundamental discoveries, more fundamental than the Univalence Axiom.

In order to prove soundness of the Univalent Foundations, it was natural for Voevodsky to consider interpreting the formal language in a model based on topological spaces. In the model, a type $X$ is interpreted as a "continuous" family of spaces (fibration) over the space parametrizing the possible values for the variables in the current context and an element $x$ of $X$ is interpreted as a section of that fibration. A universe is interpreted as the base of the universal fibration with small fibers, i.e., as the space of all spaces that are small, relative to a chosen Grothendieck universe. In 2006 Voevodsky built on an idea of Bousfield to establish an interpretation of univalence in the model, showing that the space of homotopy equivalences between small spaces $X$ and $Y$ is naturally homotopy equivalent to the space of paths between the corresponding points in the space of all small spaces. That topological statement of univalence is both appealing and plausible, but doesn't seem to have been previously noticed.

Normally the invocation of an axiom in a proof destroys the possibility to interpret the proof as a computation, but Voevodsky conjectured that, for the Univalence Axiom, computation could be accomplished, anyway. Coquand has devised a new formal language called cubical type theory [Cohen, Coquand, Huber, \& Mörtberg] in which univalence is a theorem, rather than an axiom, thereby restoring computability and justifying the soundness of axiom-free proofs without assuming the existence of Grothendieck universes in ZFC.

Interestingly, Voevodsky didn't see how to impose univalence on equality types until late 2009, even though he had proved the corresponding topological statement in 2006, saying later that: "I did not know how to model the Martin-Löf identity types and thought that different identity types will need to be introduced that will satisfy univalence", and that "I was hypnotized by the mantra that the only inhabitant of the [identity] type is reflexivity, which made them useless from my point of view".

Voevodsky suffered from long periods of depression, during which his mathematical productivity was greatly diminished. In 2013 he recovered from his final such period, and thereafter he was highly productive, posting 15 preprints to arXiv.org before his death; others remain in an unfinished 
state in his files. One strategy he developed during that time for maintaining a high level of productivity was to designate alternate days as the only days when he would work on mathematics. Approach him with a mathematical question on a nonmath day, and you would most likely be asked to wait a day to pose it.

The aim of most of those final articles is to establish the soundness of the Univalent Foundations, as well as of similar such formal systems, by developing a complete mathematical theory of type theories. It's a formidable task, because the entire system, with all of its inference rules and equations, has to be considered as a complete entity: it doesn't suffice to consider one rule at a time. He isolated the key step of the proof as his "Initiality Conjecture" and intended, perhaps, to prove it in the last article of the series, which remains unwritten.

His ability to separate a large project, such as this one, into manageable portions which could be completed sequentially, was part of what made him so productive, and it was also on display during his work on motivic homotopy theory.

The ideas in Voevodsky's Foundations repository form the basis of several formalization projects in homotopy type theory, and the code itself lives on as part of the UniMath repository, started as a collaboration to formalize a substantial body of mathematics. Eventually formalizing his work in motivic homotopy theory would be a fitting memorial to Vladimir. Even better would be also to find a constructive replacement for an argument of Merkurjev and Suslin used therein that appeals to the axiom of choice, for he has stated that "solving this problem would be very beneficial for the whole field".

DANIEL R. GRAYSON 